\title{
Our panel of experts highlight the most important research articles across the spectrum of topics relevant to the field of pain management
}

Expert panel: Nanna Finnerup, Danish Pain Research Center, Aarhus University, Denmark; Michael A Erdek, Johns Hopkins University, MD, USA

\section{Paterson K, Lolignier S, Wood JN, McMahon SB, Bennett DL. Botulinum toxin-a treatment reduces human mechanical pain sensitivity and mechanotransduction. Ann. Neurol. 75(4), 591-596 (2014).} Botulinum toxin type A (BTX-A) has recently been used to treat headache and neuropathic pain, but the mechanisms for this analgesic effect is not completely understood. This study examined the modality-specific effects of intradermal injection of BTX-A. Quantitative sensory testing and sudomotor function was tested after chemical challenge with histamine and alkyl isothiocyanate and BTX-A treatment in healthy subjects. In addition, the effect of BTX-A on neuronal excitability and mechanotransduction was assessed in cultured dorsal root ganglion neurons from mice. BTX-A had a delayed and chronic effect and reduced itch, pain and neuroinflammation in response to chemical challenges. BTX-A reduced the sensitivity to noxious mechanical stimuli but had no effect on thermal sensitivity. Consistent with this, BTX-A decreased the proportion of neurons expressing slowly adapting mechanically gated currents linked to mechanical pain transduction while it had no effect on sensory neuronal excitability. This study thus suggests that BTX-A acts by inhibiting mechanotransduction, a finding that has clinical implications as it suggest that patients with mechanical hypersensitivity may be more likely to respond to treatment with BTX-A.

- Written by Nanna Finnerup

Ulirsch JC, Ballina LE, Soward AC

et al. Pain and somatic symptoms are sequelae of sexual assault: results of a prospective longitudinal study. Eur. J. Pain 18(4), 559-66 (2014).

Persistent widespread pain and symptoms such as nausea, difficulty concentrating and fatigue are sometimes seen after trauma without evidence of major tissue damage, and the mechanisms are often unknown. This prospective study found that widespread pain and somatic symptoms were common sequelae after sexual assault. The study followed women who presented for care within $48 \mathrm{~h}$ of sexual assault with four interviews over 3 months. Clinically significant pain was common with no association between pain symptoms in a body region and direct trauma to the region. The pain was often widespread and more than $10 \%$ of women at each time point reported clinically significant worsening pain in five or more body regions. In addition, somatic symptoms, such as headache, nausea, restlessness, fatigue and difficulty sleeping increased. Further studies investigating persistent widespread musculoskeletal pain and somatic symptoms after sexual assault and traumas, such as motor vehicle collision, and the relation to stress,

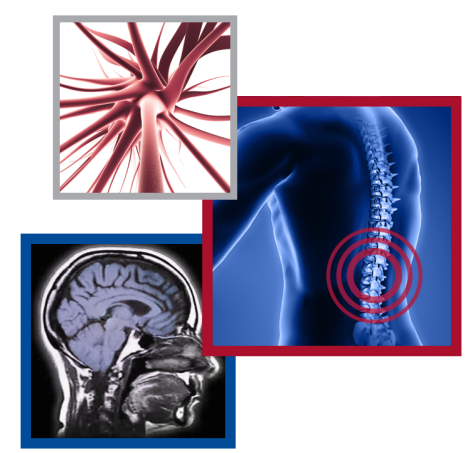

News \& Views

News

Journal Watch
Future 
pre-existing characteristics and other possible factors, are needed.

- Written by Nanna Finnerup

\section{Sachs AJ, Babu H, Su Y et al. Lack of efficacy of motor cortex stimulation for the treatment of neuropathic pain in 14 patients. Neuromodulation doi:10.111/ ner.12181 (2014) (Epub ahead of print).}

A retrospective record review of 14 patients having undergone motor cortex stimulation over an 8 -year period for intractable neuropathic pain refractory to medical management was carried out. Six of these patients suffered from trigeminal pain and had undergone previous unsuccessful ablative procedures. Implantation of the device was undertaken without benefit of a trial using MRI guidance, intraoperative somatosensory evoked potentials and evaluation of motor response to stimulation. Leads were positioned such that one array lay over the motor cortex and one over the primary somatosensory cortex or central sulcus. Postoperative imaging was obtained to further correlate placement with outcomes. A systematic sequence of changing amplitude, frequency and pulse width of the paddle lead 8-contact configuration was carried out to optimize relief. Five of the 14 patients exhibited initial relief of $>50 \%$ judged by visual analog scale score, but in only two of these was the relief maintained over the average postoperative follow-up period of 55.5 weeks. Twelve of the 14 patients underwent postoperative imaging, and in all, 12-lead placement was deemed appropriate. Within the limits of this small, retrospective study, motor cortex stimulation failed to produce adequate long-term pain relief in this patient population.

- Written by Michael Erdek

Arle JE, Carlson KW, Mei L et al. Mechanism of dorsal column stimulation to treat neuropathic but not nociceptive pain: analysis with a computational model.
Neuromodulation doi:10.1111/ner.12178 (2014) (Epub ahead of print).

A computational model of the human spinal cord was created to involve some 360,000 individual neurons and dendritic processing of some $60 \mathrm{mil}-$ lion synapses. The investigators created both neuropathic and nociceptive signals via activation of topographically isolated regions of excitatory interneurons and high-threshold nociceptive fibers to drive analogous regions of wide dynamicrange neurons. Subsequent to this, dorsal column activity was created at levels deemed clinically appropriate $(\mathrm{A} \beta$ firing rate $0-110 \mathrm{~Hz}, 210 \mu \mathrm{sec}$ pulse width, $50-100 \mathrm{~Hz}$ frequency, $1-3 \mathrm{~V}$ amplitude). Ultimate circuit analysis yielded results in contradistinction to classic gate control theory, concluding that nociceptive and neuropathic signaling are distinct and that dorsal column stimulation has its predominant effect on neuropathic signaling only. The inhibition of the neuropathic signal was deemed to be due to inhibition of centrally sensitized pathological neuron groups and, in turn, the wide dynamic-range pain transmission cells. Thus the classic gate-control theory is inadequate in its failure to account for central sensitization found in neuropathic pain as shown by this neural circuitry simulation model. A potential shortcoming of this computational model is its failure to account for descending inhibition connections from the brain.

- Written by Michael Erdek

Financial \& competing interests disclosure

$N$ Finnerup receives research funding from the EUROPAIN Investigational Medicines Initiative, which is a publicprivate partnership between the European Federation of Pharmaceutical Industries and Associations (EFPIA) and EU. N Finnerup has also received honoraria from Pfizer and Grünenthal. The authors have no other relevant affiliations or financial involvement with any organization or entity with a financial interest in or financial conflict with the subject matter or materials discussed in the manuscript apart from those disclosed.

No writing assistance was utilized in the production of this manuscript. 